\title{
Comunicación y Sociedad: aportes y sesgos en el campo académico de la comunicación en México
}

\section{Raúl Fuentes NaVARro*}

\begin{abstract}
Los indicadores más importantes de la organización social en un área de investigación son las discusiones informales de investigación, las colaboraciones publicadas, las relaciones con profesores y la influencia de colegas en la selección de problemas y técnicas de investigación

Diana Crane
\end{abstract}

Este artículo despliega un análisis descriptivo de los cuarenta primeros números de la revista Comunicación y Sociedad, para resaltar las relaciones de su contenido con el proyecto académico del Departamento de Estudios de la Comunicación Social, que la edita desde 1987, y con la producción del campo académico de la investigación de la comunicación en México. Su objetivo es renovar la información disponible y generar nuevas preguntas a propósito del espacio social en que se produce conocimiento en el país sobre las múltiples y complejas relaciones entre la comunicación y la sociedad.
A descriptive analysis is displayed in this article, concerning the first forty issues of Comunicación y Sociedad, focusing on the relationships between the contents of the journal and the general academic project of its editing institution, the Department of Social Communication Studies, as well as with the major trends on the field of communication research in Mexico. The aim of the article is to refresh information in order to ask new questions on the social space in which knowledge on the variated and complex relations among communication and society is being produced in Mexico.

* Departamento de Estudios de la Comunicación Social de la Universidad de Guadalajara y Departamento de estudios Socioculturales del Instituto Tecnológico y Estudios Superiores de Occidente. 
La publicación, a fines de 2003, del número 40 de Comunicación y Sociedad (con julio-diciembre de 2001 como fecha de portada) marcó el cierre de una época para la revista y el inicio de otra, en que se renuevan los propósitos y se asumen los aprendizajes colectivos acumulados a partir de 1987 en el Departamento de Estudios de la Comunicación Social (DECS) de la Universidad de Guadalajara (UdeG). Este cambio de época, debido a razones diversas, es ocasión propicia para la revisión del trayecto recorrido y para la elaboración de un "estado de la cuestión" actualizado sobre los objetivos y realizaciones del proyecto editorial que ha orientado la publicación durante más de 15 años.

La intención de este trabajo es contribuir a esa revisión aportando datos sistematizados sobre los textos que han sido publicados en la revista y la propuesta de algunas relaciones de estos escritos con los contextos académicos y sociales en que se han producido y puesto en circulación. No se trata de proponer conclusiones o juicios sobre la propia revista sino de facilitar la interpretación y la discusión de algunas de las condiciones y de las tendencias más significativas que su presencia ha planteado en el campo académico de la comunicación en México. Es decir, se busca renovar la información disponible y generar nuevas preguntas a propósito del espacio social en que se produce conocimiento en el país sobre las múltiples y complejas relaciones entre la comunicación y la sociedad.

A pesar de que no son muy abundantes los estudios sistemáticos sobre las publicaciones académicas, se dispone de varios artículos referidos al campo de la comunicación, que son antecedentes cercanos de éste, como los firmados por Francisco Aceves (1993), Raúl Fuentes (1990, 1994, 1996a, 1998a, 2000), Héctor Gómez Vargas (2003), Guillermo Orozco (2000), Florencia Saintout (1998) y Eduardo Scheffler (1998), así como la tesis de maestría de Karla Margarita Ramírez (2003), premiada por el Consejo Nacional para la Enseñanza y la Investigación de las Ciencias de la Comunicación (CONEICC). También, para este caso, es importante recuperar el análisis realizado por Teresa Tovar (1997), en ocasión del décimo aniversario de Comunicación y Sociedad.

\section{EL PROYECTO FUNDADOR Y SUS SECUELAS EDITORIALES}

Pablo Arredondo, al exponer los procesos y propósitos que llevaron a la Universidad de Guadalajara a abrir en 1986 el Centro de Estudios de la Infor- 
mación y la Comunicación (CEIC) -transformado en 1994 en el Departamento de Estudios de la Comunicación Social-, cita entre los objetivos fundamentales formulados en la propuesta original, el de "convertirse, dentro del Occidente nacional, en un centro de difusión del conocimiento científico de las disciplinas que inciden en la comunicación, por medio de la realización de eventos académicos y la producción de materiales pertinentes" (Arredondo 1997: 18). El proyecto se ubicaba con mucha claridad en un diagnóstico agudo del campo académico en el que se insertaría:

El CEIC fue concebido como una alternativa académica de avanzada en donde se llevaría a cabo lo que escasamente se realizaba en otras universidades. Los pilares en que se sostendría serían cuatro: la investigación, la educación de posgrado y especializada, la difusión científica y la vinculación académica (Arredondo 1997: 19).

Comunicación y Sociedad apareció bajo la forma de "Cuadernos del CEIC" con tres números monográficos publicados en 1987: La televisión en Guadalajara; génesis y desarrollo, de Francisco de Jesús Aceves; Televisión y producción de significados (tres ensayos), de Guillermo Orozco Gómez, y Centralización, poder y comunicación en México, de Enrique E. Sánchez Ruiz. Muy pronto, el proyecto evolucionó:

[...] en sus inicios, Comunicación y Sociedad pretendió ser una publicación restringida a la difusión de los avances de investigación y reflexión teórica de los investigadores... Sin embargo, viendo la demanda de colaboradores, el interés de los lectores, la escasez de publicaciones de corte académico y la necesidad de abrir nuevos medios de difusión de conocimiento en el área de la comunicación social, se decidió publicarla periódicamente (Tovar 1997: 8).

Así, a partir del número 8 (enero-abril de 1990), la revista adoptó una periodicidad cuatrimestral, la cual fue modificada a semestral a partir del número 35 (enero-junio de 1999). No obstante la continuidad de la numeración hasta el 40, la colección completa consta de 33 ediciones; es decir, incluye siete "números dobles". La descripción general de esta secuencia se muestra en la Tabla 1. 
TABLA 1

EJEMPlaRes PUblicados de ComuniCaCión Y SOCIEDAD

\begin{tabular}{lll} 
Número & Periodicidad & Fecha de portada \\
1 & Monográfico & 1987 \\
2 & Monográfico & 1987 \\
3 & Monográfico & 1987 \\
$4 / 5$ & Sin periodicidad & 1989 \\
6 & Sin periodicidad & 1989 \\
7 & Sin periodicidad & 1989 \\
8 & Cuatrimestral & enero-abril 1990 \\
9 & Cuatrimestral & mayo-agosto 1990 \\
$10 / 11$ & Cuatrimestral & septiembre 1990-abril 1991 \\
12 & Cuatrimestral & mayo-agosto 1991 \\
13 & Cuatrimestral & septiembre-diciembre 1991 \\
$14 / 15$ & Cuatrimestral & enero-agosto 1992 \\
$16 / 17$ & Cuatrimestral & septiembre 1992-abril 1993 \\
$18 / 19$ & Cuatrimestral & mayo-diciembre 1993 \\
20 & Cuatrimestral & enero-abril 1994 \\
21 & Cuatrimestral & mayo-agosto 1994 \\
$22 / 23$ & Cuatrimestral & septiembre 1994-abril 1995 \\
24 & Cuatrimestral & mayo-agosto 1995 \\
$25 / 26$ & Cuatrimestral & septiembre 1995-abril 1996 \\
27 & Cuatrimestral & mayo-agosto 1996 \\
28 & Cuatrimestral & septiembre-diciembre 1996 \\
29 & Cuatrimestral & enero-abril 1997 \\
30 & Cuatrimestral & mayo-agosto 1997 \\
31 & Cuatrimestral & septiembre-diciembre 1997 \\
32 & Cuatrimestral & enero-abril 1998 \\
33 & Cuatrimestral & mayo-agosto 1998 \\
34 & Cuatrimestral & septiembre-diciembre 1998 \\
35 & Semestral & enero-junio 1999 \\
36 & Semestral & julio-diciembre 1999 \\
37 & Semestral & enero-junio 2000 \\
38 & Semestral & julio-diciembre 2000 \\
39 & enero-junio 2001 \\
40 & julio-diciembre 2001 \\
\hline Femestral &
\end{tabular}

Fuente: propia. 


\section{TABLA 2}

ORIGEN DE LAS COLABORACIONES PUBLICADAS EN TRES REVISTAS ACADÉMICAS MEXICANAS (PORCENTAJES)

Local "propia institución"

Nacional "ajenos mexicanos"

Internacional "ajenos extranjeros"

Comunicación y Sociedad

Estudios sobre las Culturas Contemporáneas

41.5

27.1

31.4

Versión, estudios de comunicación y política

23.3

40.6

36.1

$38.7 \quad 16.1$

Fuente: Karla Ramírez 2003.

La conversión de unos cuadernos de difusión monográficos en una revista académica (publicación periódica con arbitraje sobre colaboraciones de distinta temática y diverso origen institucional en cada número), supuso el establecimiento de nuevas condiciones y procedimientos de producción. Esta transición es evidente desde el número 4/5, publicado en 1989, hasta el número 20, correspondiente a enero-abril de 1994, fecha en que Comunicación y Sociedad fue incorporada por el Consejo Nacional de Ciencia y Tecnología (Conacyt) al Índice de Revistas Científicas Méxicanas. Como es común en el ámbito académico mexicanoy a diferencia de los journals científicos internacionales, que son creados y mantenidos por las asociaciones científicas de las especialidades de investigación que cubren-, la revista se ubicó al mismo tiempo como un órgano de diseminación del conocimiento especializado en comunicación, y como tal en una instancia central del campo académico, y como un medio de difusión de los productos generados por los investigadores adscritos a la institución editora, es decir, como una pieza central para el cumplimiento de los propósitos particulares de un grupo de investigadores y de una universidad en concreto.

Como lo muestra el análisis de Karla Ramírez (2003), esta revista de la Universidad de Guadalajara, comparada con Estudios sobre las Culturas Contemporáneas de la Universidad de Colima y con Versión, estudios de comunicación y política de la Universidad Autónoma Metropolitana Xochimilco, alcanza la proporción más alta de textos producidos por los investigadores "propios" sobre los "ajenos", es decir, adscritos a otras instituciones, nacionales o extranjeras. 
Esta comparación, que sería interesante extender a otras publicaciones, sugiere que a lo largo de su trayectoria como publicación académica, Comunicación y Sociedad ha logrado conciliar, de una manera más equilibrada que otras revistas, la doble función de servir a la difusión de los productos de la investigación realizada en la institución que la edita, y en el campo académico en que se ubica. Habría que recordar aquí la tensión que Burton R. Clark (1992) considera como constitutiva del trabajo académico: la que surge del hecho de que los investigadores "pertenecen", al mismo tiempo, a una institución o "establecimiento" multidisciplinario y a un campo o "disciplina" transinstitucional. Por ello resulta importante recuperar la manera en que sus fundadores concibieron la investigación en el ceIc: según se ha dicho ya, como uno de los "pilares" del proyecto.

El primero, y más importante, de los pilares (la investigación), supuso una visión de carácter "multidisciplinario" a través del cual se analizarían los fenómenos comunicativos considerando sus más obvias implicaciones para la sociedad. Las relaciones entre la comunicación y el poder político, entre la comunicación y la economía, y entre la comunicación y los fenómenos culturales, sirvieron de guía para formar tres programas de investigación dentro de los que se aglutinarían proyectos específicos. Los ejes temáticos de la investigación se establecieron con márgenes amplios, en vista de la escasa especialización que se percibía en el campo académico de la comunicación. Los programas de investigación fueron vistos como grandes avenidas por las que deberían circular los proyectos especializados (Arredondo 1997: 19-20).

Si bien esa división tripartita de las líneas o "programas" de investigación fue modificada con el transcurso de los años para adecuar la organización del trabajo a las necesidades institucionales y al crecimiento del número de investigadores -sobre todo a partir de la departamentalización en 1994-, conviene recuperarla como esquema de reconocimiento de los constitutivos de esa "multidisciplinariedad" inherente al campo de la comunicación. De esta manera se puede también facilitar la contrastación de las tendencias encontradas en Comunicación y Sociedad con las detectadas en una escala más amplia: la de la investigación académica de la comunicación en México, como se hizo en su sistematización más reciente: 
La división clásica de los estudios sociales en dimensiones económicas, políticas y culturales sigue siendo relativamente útil, si bien es indispensable reconocer que los objetos de investigación de la comunicación, en particular los referidos a los medios, suelen cruzar las tres esferas, lo cual no impide que los análisis particulares enfaticen los factores correspondientes a alguna de ellas $[\ldots]$ no puede decirse que en los años más recientes prevalezca en la investigación mexicana de la comunicación una tendencia a "economizarla", "politizarla" o "culturizarla", sino que hay desarrollos que se proyectan sobre las tres esferas (Fuentes 2003: 25).

Un análisis de los textos publicados en Comunicación y Sociedad, intentando una categorización temática orientada por criterios como el aquí apuntado, puede servir para explorar las manifestaciones concretas, en esta publicación, de las tendencias detectadas para el campo en su conjunto nacional, así como las líneas de trabajo que han generado aportes más significativos por parte de los académicos adscritos a la Universidad de Guadalajara y algunas de las articulaciones que se han construido entre ellos y otros investigadores, nacionales y extranjeros. De esta manera podrían apreciarse mejor los "sesgos", es decir, las orientaciones preferentes, que la revista introduce o refuerza en el campo académico en que se le ha reconocido como la publicación periódica más importante (Fuentes 1998b: 222).

\section{UN ANÁLISIS BIBLIOMÉTRICO DE AUTORES, TEMAS Y ENFOQUES}

En cuanto a su formato editorial, Comunicación y Sociedad ha publicado textos de diverso carácter: en todos los números hay una "presentación"; en algunos, una distinción entre secciones de "artículos" y "ensayos"; ocasionalmente "testimonios", "crónicas", "entrevistas" y "debates"; y con mayor frecuencia, aunque no en todos los números, "reseñas" y "materiales para el estudio de los medios". Para efectos de esta sistematización, se excluyen las presentaciones y las reseñas y se tratan indistintamente los textos restantes, como productos de y contribuciones a la investigación de la comunicación. En consecuencia, una primera concentración de datos sobre el contenido de la revista permite apreciar la composición de los 253 textos publicados en relación con su origen institucional, indicado por la adscripción del autor. 


\section{TABLA 3}

Textos PUblicados en Comunicación Y SOCIEDAD SEGÚn ADSCRIPCIÓN DE SUS AUTORES

Número “Local Nacional “, “nternacional
"propia institución" "ajenos mexicanos" "ajenos extranjeros"

\begin{tabular}{|c|c|c|c|}
\hline 1 & 1 & 0 & 0 \\
\hline 2 & 0 & 1 & 0 \\
\hline 3 & 1 & 0 & 0 \\
\hline $4 / 5$ & 5 & 1 & 0 \\
\hline 6 & 3 & 0 & 0 \\
\hline 7 & 2 & 1 & 0 \\
\hline 8 & 4 & 1 & 0 \\
\hline 9 & 1 & 3 & 2 \\
\hline 10/11 & 3 & 4 & 3 \\
\hline 12 & 2 & 5 & 2 \\
\hline 13 & 3 & 4 & 3 \\
\hline $14 / 15$ & 5 & 4 & 4 \\
\hline $16 / 17$ & 4 & 1 & 3 \\
\hline $18 / 19$ & 3 & 4 & 5 \\
\hline 20 & 5 & 3 & 1 \\
\hline 21 & 2 & 0 & 7 \\
\hline $22 / 23$ & 3 & 1 & 1 \\
\hline 24 & 3 & 2 & 3 \\
\hline $25 / 26$ & 6 & 3 & 2 \\
\hline 27 & 5 & 3 & 1 \\
\hline 28 & 5 & 2 & 1 \\
\hline 29 & 2 & 1 & 6 \\
\hline 30 & 8 & 5 & 1 \\
\hline 31 & 3 & 2 & 5 \\
\hline 32 & 3 & 0 & 5 \\
\hline 33 & 3 & 3 & 1 \\
\hline 34 & 3 & 1 & 4 \\
\hline 35 & 1 & 3 & 5 \\
\hline 36 & 2 & 3 & 2 \\
\hline 37 & 4 & 1 & 5 \\
\hline 38 & 1 & 4 & 5 \\
\hline 39 & 1 & 3 & 2 \\
\hline 40 & 2 & 3 & 3 \\
\hline Total: 253 & $99(39.1 \%)$ & $72(28.5 \%)$ & $82(32.4 \%)$ \\
\hline
\end{tabular}

Fuente: propia. 
Es de hacerse notar que entre la Tabla 3 y la Tabla 2 hay una leve discrepancia en los porcentajes señalados para el origen institucional de los textos publicados en Comunicación y Sociedad. Esta discrepancia se debe a que Ramírez (2003) incluyó en su trabajo los 229 textos publicados hasta el número 37, y que de los 24 artículos correspondientes a los tres últimos números, sólo cuatro corresponden a académicos de la Universidad de Guadalajara. No obstante, esta recomposición de los porcentajes incrementa aún más la tendencia al equilibrio señalada antes, en cuanto al origen de los trabajos.

Como sería de esperar, los autores individuales que con mayor frecuencia han contribuido con textos publicados en la revista, están o han estado adscritos al DECS. Debido a que a lo largo de los años se incorporaron al departamento varios investigadores que, desde su adscripción institucional anterior habían sido ya autores de textos publicados en la revista, se enlista en la Tabla 4 a los autores con mayor número de artículos incluidos, manteniendo sólo la distinción entre los que trabajan en instituciones mexicanas y los que lo hacen en el extranjero. De esta manera, se puede ilustrar al mismo tiempo otro factor: la alta correspondencia entre las frecuencias altas de publicación (mayores de tres) en la revista y la pertenencia al Sistema Nacional de Investigadores (SNI).

Puede verse que 15 de los 19 autores de tres o más textos publicados en Comunicación y Sociedad pertenecían en 2003 al Sistema Nacional de Investigadores, dos de ellos en el máximo nivel y siete en el nivel II. En otros términos, la Tabla 4 indica que 90 textos de los 171 de origen nacional (52.6\% de ellos), provienen de investigadores oficialmente reconocidos y distinguidos, y que la mitad de esos textos pertenecen a académicos actualmente activos en el DECS. Hay que subrayar también que, aunque en una proporción mucho menor, se han publicado artículos firmados por investigadores más jóvenes, en proceso de formación. La revista muestra, en este sentido, evidencias claras de ser mucho más un órgano de diseminación del trabajo de los especialistas más reconocidos, que un espacio de estímulo a la expansión y reproducción del campo académico.

Entre los investigadores extranjeros que han colaborado con textos en la revista, ocho lo han hecho en más de una ocasión: la brasileña Maria Immacolata Vassallo de Lopes (con tres); los españoles Marcial Murciano y Enrique Bustamante, los brasileños José Marques de Melo y Nilda Jacks, el canadiense François Demers, el danés Thomas Tufte y 
TABLA 4

AUTORES NACIONALES CON MAYOR NÚMERO DE TEXTOS PUBLICADOS EN COMUNICACIÓN Y SOCIEDAD Y NIVEL EN EL SNI EN 2003

\begin{tabular}{lll} 
Investigador(a) & Textos & SNI \\
Enrique E. Sánchez Ruiz (UdeG) & 15 & III \\
Francisco de Jesús Aceves González (UdeG) & 10 & I \\
Guillermo Orozco Gómez (UIA-UdeG) & 8 & III \\
Raúl Fuentes Navarro (ITESo-UdeG) & 8 & II \\
Cecilia Cervantes Barba (UdeG) & 8 & - \\
Gilberto Fregoso Peralta (UdeG) & 7 & - \\
Martha Renero Quintanar (UIA-UdeG) & 6 & I \\
Javier Esteinou Madrid (UAM-X) & 6 & I \\
Sarah Corona Berkin (UAM-X-UdeG) & 5 & II \\
Rossana Reguillo Cruz (ITESO-UdeG) & 5 & II \\
Delia Ma. Crovi Druetta (UNAM) & 5 & II \\
Celia del Palacio Montiel (UdeG) & 4 & I \\
Francisco Hernández Lomelí (UdeG) & 4 & C \\
Armando Martín Ibarra López (UdeG) & 4 & - \\
José Carlos Lozano Rendón (ITESM) & 4 & I \\
Raúl Trejo Delarbre (IIS UNAM) & 4 & II \\
María Elena Hernández Ramírez (UdeG) & 3 & - \\
Eduardo de la Vega Alfaro (UdeG) & 3 & II \\
Renée de la Torre Castellanos (CIESAS) & 3 & II \\
\hline
\end{tabular}

Fuente: propia.

el británico John B. Thompson (con dos cada uno). En total, además de México, el origen de los textos publicados en Comunicación y Sociedad remite a 18 países americanos, europeos y asiáticos.

Para el análisis de los temas de los textos, debe enfrentarse el problema de las categorías de clasificación, que no ha sido posible hasta la fecha unificar o establecer con un mínimo de consistencia. Esta condición, además de ser reveladora de la "desarticulación múltiple" que caracteriza al campo desde su origen (Fuentes 1998b), es también una consecuencia de las maneras en que se ha desarrollado. Al introducir un análisis de tendencias temáticas y de contenido de los productos mexicanos de investigación de la comunicación, se señala que:

[...] es cada vez más difícil delimitar las fronteras de la investigación de la comunicación con otras áreas temáticas y metodológicas de las ciencias sociales y las humanidades. Además de la creciente búsqueda de la 
interdisciplinariedad, es necesario considerar que, sobre todo en el periodo más reciente, el entorno económico-político y sociocultural de la comunicación en México y en el mundo ha sido sometido a transformaciones aceleradas y en apariencia radicales en casi todos los ámbitos. Se puede hablar, así, de la incidencia de factores internos y externos al campo académico para dar cuenta de las reconfiguraciones de la investigación que están en proceso. Así, en los documentos sistematizados se evidencia una diversificación temática muy amplia, que apunta al mismo tiempo hacia muchos ámbitos y dimensiones sociales y hacia muy diversos campos disciplinarios. La clasificación de los documentos, utilizando categorías mutuamente excluyentes sobre ejes temáticos determinados, se dificulta hasta un grado insostenible (Fuentes 2003: 22-23).

Al igual que en la sistematización documental más reciente de la producción académica del campo, en Comunicación y Sociedad "se constata que ante el entorno cambiante e incierto donde se ubican sus objetos de estudio, los agentes académicos de la investigación han agudizado su atención crítica sobre el propio campo, tanto en su dimensión institucional (universitaria) como en cuanto a su configuración científica" (Fuentes 2003: 23). Cuarenta y cinco artículos (17.8\%) del total de los publicados en la revista tienen como objeto principal de análisis el propio campo, sea en su dimensión institucional, en su dimensión teórico-metodológica o en las tendencias empíricas de su producción, además de que la reflexión metodológica y la exposición o discusión de técnicas de investigación de diversa índole está presente en muchos de los artículos que tratan primordialmente otros temas, sean "medios", "sujetos" o "escenarios" de comunicación. Es muestra emblemática, aunque ni con mucho única, de esta atención sistemática a las condiciones del propio campo, el número 30, conmemorativo del décimo aniversario de la revista, en que según la "presentación", el comité editorial se propuso reunir, "por una parte, artículos que revisaran el estado de la investigación en comunicación en la última década desde diferentes puntos de vista, y por otra, artículos que señalaran nuevas propuestas y acercamientos teórico-metodológicos a las relaciones entre comunicación y sociedad" ( $C y S$ núm. 30: 8).

$\mathrm{Al}$ igual que en la mayor parte de las instituciones donde se ha cultivado la investigación de la "comunicación" (que se entiende restringida, aun sin necesidad de especificarlo, a sus modalidades "social", "colectiva" o "pública", y no a las "interpersonales", "grupales" o "privadas"), en la Universidad de Guadalajara se ha privilegiado el análisis 
de los "medios", que sólo puede desarrollarse multidisciplinariamente (Sánchez Ruiz 1992). Esta delimitación del objeto y condición epistemológica, constitutivas del campo mismo, se manifiestan con claridad en las páginas de Comunicación y Sociedad. En la Tabla 5 se desglosan los temas generales de los textos publicados en la revista, según su referencia a los medios o a otros objetos de análisis a lo largo del tiempo. Se trata, con ello, de documentar más finamente lo que Enrique E. Sánchez Ruiz sintetiza de una manera muy clara:

De hecho, la investigación mexicana de la comunicación ha sido muy poco investigación de la comunicación, y más de los medios. Y éstos han sido analizados, más que como instancias sociales productoras de sentido, predominantemente en tanto instituciones sociopolíticas. En la medida en que los medios, las industrias culturales y todo el complejo tecnológico del entretenimiento y la información en que han devenido, insertos a su vez en los procesos globalizadores actuales, son de hecho procesos complejos, multidimensionales, su estudio ha requerido, requiere y seguirá requiriendo el recurso a las ciencias y campos sociales y humanísticos necesarios para en cada caso comprender cada una de esas dimensiones y sus posibles articulaciones con otras (Sánchez Ruiz 1997: 60).

Los resultados de esta clasificación indican que casi dos tercios (64.4\%) de los textos publicados en Comunicación y Sociedad enfocan prioritariamente el análisis de una o varias dimensiones de los "medios". El tercio restante, en proporciones idénticas, se orienta al conocimiento del propio campo de investigación por una parte y, por otra, a la apertura o complementariedad de objetos de estudio "no-mediáticos", emergentes o asociados de diversas maneras multidisciplinarias al análisis de las relaciones entre "comunicación" y "sociedad".

Aunque sin el empleo de categorías mutuamente excluyentes como las empleadas en la confección de la Tabla 5, las dos sistematizaciones documentales más recientes (Fuentes 1996b; 2003) de la investigación de la comunicación en México, correspondientes a los periodos 19861994 y $1995-2001$, indican que $71.3 \%$ y $73.4 \%$ de los documentos incluidos respectivamente, hacen referencia a los medios de comunicación como sus objetos de estudio, mientras que al campo académico lo hacen $13.2 \%$ y $20.3 \%$, respectivamente. Sin poderse comparar estrictamente los datos por la forma en que fueron confeccionados, queda suficientemente ilustrada la correspondencia aproximada entre la revista y el campo del que forma parte en cuanto al predominio de los medios 


\section{TABLA 5}

Textos publicados en Comunicación y Sociedad SEgún temas de REFERENCIA PRIMORDIAL A "MEDIOS" U OTROS OBJETOS

$\begin{array}{llllllllll}A & B & C & D & E & F & G & H & I & J\end{array}$

$40 \quad 3$

Total $45 \quad 33$

$$
8 \quad 9
$$

$9-56$

$\mathrm{A}=$ Número; $\mathrm{B}=\mathrm{Campo}$ académico; $\mathrm{C}=$ Prensa e impresos; $\mathrm{D}=\mathrm{C}$ ine; $\mathrm{E}=$ Radio; $\mathrm{F}=$ Televisión y video; $\mathrm{G}=$ Internet y digitales; $\mathrm{H}=$ Medios en conjunto; $\mathrm{I}=\mathrm{Otros}$ objetos; J=Medios / Total

Fuente: propia. 
como objetos privilegiados de estudio, a la proporción en que se manifiesta la atención a temas de comunicación no-mediática, y en cuanto a la creciente reflexión sobre las condiciones en que se realiza y los recursos científicos que utiliza la investigación.

Entre los medios, los más estudiados siguen siendo, como es de esperar, los audiovisuales y, entre éstos, sobre todo la televisión. Le siguen en frecuencia los "medios en conjunto" y los impresos, tanto en Comunicación y Sociedad como en el campo más amplio, donde también permanecen como minoritaria pero constantemente presentes los estudios sobre cine y radio. De la misma manera, los medios digitales de telecomunicación, incluyendo la desafortunada denominación de "nuevas tecnologías" y más recientemente Internet, son objetos de atención en rápido crecimiento. Por ello no se encuentra justificación para profundizar más en la comparación entre los temas de la revista y los del campo desde esta óptica, pues queda suficientemente ilustrada la correspondencia general ya indicada. Es más interesante, en este sentido, explorar algunas particularidades de Comunicación y Sociedad que pueden interpretarse en el sentido de los aportes y los sesgos que su presencia ha ofrecido al campo.

Una primera aproximación consiste en clasificar los 163 textos que tienen como tema principal a los medios, en relación con el tipo de abordaje que presentan sobre ellos: bien sea desde la "fase" del proceso de la comunicación que privilegian, o desde la "perspectiva" social desde la que los sitúan. Esta clasificación se presenta en la Tabla 6.

Se detecta, así, un relativo balance o una gama amplia de abordajes temáticos en los estudios de medios publicados en Comunicación y Sociedad, en donde éstos son analizados en diferentes dimensiones y desde diversas perspectivas, cada una de ellas asociada, por supuesto, a distintos métodos. Llaman la atención los vacíos: no hay estudios sobre la "institucionalidad" de la prensa o la industria editorial más en general, y tampoco sobre la circulación o distribución de impresos; además no los hay sobre la "circulación" ni sobre la "significación" de los productos radiofónicos. Sobre todos los demás tipos de abordaje acerca de los medios hay al menos un ejemplar. En esta clasificación se han incluido ocho artículos sobre el "estado de la investigación" acerca de los medios, que también hubieran podido sumarse a los textos de análisis del campo académico pero, dado que están más enfocados a dar cuenta del estado del conocimiento específico sobre los medios respectivos (todos, excepto el cine) mediante una revisión bibliográfica o una propues- 


\section{TABLA 6}

Textos publicados sobre Medios en ComUNiCACión Y Sociedad SEGÚN SU ABORDAJE TEMÁTICO

$\begin{array}{lrrrrrrr} & A & B & C & D & E & F & G \\ \text { Institucionalidad } & - & 1 & 2 & 13 & 3 & 10 & 29 \\ \text { Emisión/producción } & 7 & 2 & 2 & 2 & 2 & 3 & 18 \\ \text { Circulación/distribución } & - & 1 & - & 7 & 1 & 2 & 11 \\ \text { Recepción/consumo } & 4 & 1 & 2 & 15 & 3 & 8 & 33 \\ \text { Significación/contenido } & 12 & 2 & & 10 & 1 & 8 & 33 \\ \text { Historia/políticas/regulación } & 8 & 1 & 2 & 6 & 3 & 11 & 31 \\ \text { Estado de la investigación } & 2 & - & 1 & 3 & 1 & 1 & 8 \\ \text { Total: } & 33 & 8 & 9 & 56 & 14 & 43 & 163\end{array}$

$\mathrm{A}=$ Prensa e impresos; $\mathrm{B}=$ Cine; $\mathrm{C}=$ Radio; $\mathrm{D}=$ Televisión y video; $\mathrm{E}=$ Internet $\mathrm{y}$ digitales; $\mathrm{F}=$ Medios en conjunto; $\mathrm{G}=$ Textos Fuente: propia.

ta metodológica, se decidió incorporarlos como aportes al conocimiento de la "comunicación mediática".

Pero los textos también pueden ser clasificados según el predominio en ellos de factores correspondientes a dimensiones socioeconómicas, sociopolíticas o socioculturales en la construcción de sus objetos de investigación. De acuerdo tanto con los "programas de investigación" trazados en el proyecto fundacional del ceIc en la Universidad de Guadalajara, como con las categorías de sistematización temática empleadas en el recuento de publicaciones generadas en el campo académico mexicano en los últimos años, según se refirió más atrás, la Tabla 7 presenta una distribución de los artículos contenidos en Comunicación y Sociedad según estos grandes bloques temáticos, y el referido al campo académico. Para ello, y buscando mayor claridad, se determinan los temas típicos o más frecuentes en el campo en cada una de las "dimensiones" y se asignan a los textos de la revista de manera que resulten mutuamente excluyentes, aunque en muchos casos la inclusión pueda parecer forzada. Estos "temas típicos" tienen la pretensión de indicar las articulaciones interdisciplinarias (implícitas o explícitas en los textos), que los estudios sobre la comunicación mantienen con la investigación social.

Esta clasificación de los textos publicados en Comunicación y Sociedad, quizá por el empleo de categorías mutuamente excluyentes, arroja una composición distinta a la de la sistematización documental 19952001 (Fuentes 2003), donde los tres "entornos sociales" presentan una 


\section{TABLA 7}

TeXtos publicados en Comunicación y Sociedad SEGún SU REFERENCIA TEMÁTICA SOCIAL "TÍPICA"

Temas "típicos"

Textos

- Entorno socioeconómico

$65(25.7 \%)$

Industrias culturales, sistemas de medios.

Globalización, bloques regionales.

$33(13.0 \%)$

Modernidad, estructura y cambio social.

$5(2.0 \%)$

$9(3.6 \%)$

Sociedad de la Información, cambio tecnológico.

$18(7.1 \%)$

- Entorno sociopolítico $39(15.4 \%)$

Régimen jurídico y legislación.

$5(2.0 \%)$

Democracia y democratización.

Poder, agentes políticos, Estado y gobierno.

$11(4.3 \%)$

Partidos políticos y procesos electorales.

$8(3.2 \%)$

- Entorno sociocultural $104(41.1 \%)$

Sujetos e identidades sociales.

Cultura urbana y vida cotidiana.

$22(8.7 \%)$

Educación, ideología y creencias.

$19(7.5 \%)$

Discurso y representaciones simbólicas.

$40(15.8 \%)$

- Campo académico $45(17.8 \%)$

Epistemología, teoría, metodología.

Institucionalización, producción académica.

$24(9.5 \%)$

- Total

$253 \quad 100 \%$

Fuente: propia.

atención mucho más homogénea, sin ese tipo de categorías. A reserva de realizar una revisión más a fondo de esta discrepancia, cabe por ahora explorar lo que sugiere la Tabla 7 sobre los textos contenidos en la revista.

En primer lugar, el hecho de que -si se descuentan los textos referidos al campo académico, que alcanzan $17.8 \%$ de los textos publicados-, los estudios referidos al entorno sociocultural equivalen exactamente a la suma de los referidos a los entornos socioeconómico y sociopolítico $(41.1 \%=25.7 \%+15.4 \%)$, lo que permite matizar la interpretación del dato de que el $64.4 \%$ de los artículos enfocan prioritariamente el análisis de una o varias dimensiones de los medios. Más allá de lo que se ha desglosado ya en las Tablas 5 y 6 , surge aquí la posibilidad de distinguir, por ejemplo, entre los 33 artículos (13.0\% del total), que abordan el análisis de los medios como "industrias culturales" o como sistemas institucionales -desde enfoques predominante, 
aunque no excluyentemente, socioeconómicos-, y los 40 textos $(15.8 \%$ del total), que lo hacen en relación con el discurso y las representaciones simbólicas -desde enfoques típicamente socioculturales-. En general, los temas "típicos" incluidos en el rubro sociocultural subrayan la consideración de los sujetos sociales, los esquemas culturales y los procesos de significación como constitutivos centrales de la "comunicación", mientras que los abordajes socioeconómico y sociopolítico apuntan centralmente hacia las estructuras institucionales y las relaciones sociales como objetividades históricas, también fundamentales para la comprensión de la "comunicación”. De ahí que se postule, detrás de esta propuesta de categorías, la complementariedad (pero no la confusión) de enfoques de la investigación.

A manera de hipótesis, puede decirse que la construcción de los objetos de investigación, sobre los medios y sobre la comunicación, podría estarse orientando hacia rutas teórico-metodológicas mejor definidas, y por lo tanto a relaciones más claras entre las diversas disciplinas científico-sociales y humanísticas a las que se recurre en el campo, en el contexto de la restructuración de las ciencias sociales. Y ése podría ser el principal aporte de Comunicación y Sociedad a la constitución científica de ese campo académico en que se ubica. Sin embargo, lejos del afán de establecer "conclusiones", este análisis sugiere la realización de estudios más específicamente enfocados en los datos generados y en la agudización de los debates entre los agentes académicos interesados.

\section{Algunas ARTiCUlaciones DE LA REVISTA CON EL CAMPO ACADÉMICO}

Uno de los "sesgos" que pueden detectarse en Comunicación y Sociedad respecto al campo académico, es el impulso de una atención diferenciada, pero articulable, a los factores que circunscriben a los sistemas y procesos de comunicación en el entorno social, y a los procesos y múltiples mediaciones de la producción social del sentido que acontecen en circunstancias siempre situadas. La articulación principal, desde el punto de vista estrictamente científico, no puede estar sino en el plano metodológico de la investigación, que recibe en muchos de los textos publicados en Comunicación y Sociedad una atención explícita, sea en cuanto a la revisión y desarrollo de sus condiciones propias (epistemológicas, teóricas, técnicas), como en cuanto a su empleo racional en la construcción de los diversos objetos de investigación. 
Un estudio reciente, realizado como tesis de maestría por Elizabeth Bellón (2003), permite desarrollar algunas otras hipótesis acerca de las relaciones ("aportes y sesgos") entre los textos publicados en Comunicación y Sociedad y la construcción de conocimiento científico en el campo académico de la comunicación. Una de sus premisas, de entrada, es que:

El avance del campo académico de la comunicación, sea para su "sobrevivencia" como especialidad o campo problemático de las ciencias sociales, sea para su "disolución" o "desaparición" en el territorio aparentemente más vasto de las ciencias sociales, podría articularse con la vigilancia que los investigadores realicen en torno a su oficio, "en solitario" y "en comunidad", pues los investigadores suelen avanzar a la par de su campo pero, también, podrían progresar junto con sus objetos (Bellón 2003: V).

Bellón propone, bachelardeanamente, un modelo de tres grados de "vigilancia" (sobre el objeto, sobre el método y sobre el sujeto), que "podría ser criterio de demarcación y herramienta metodológica para quienes aún pretenden construir interpretaciones 'verdaderas' sobre fragmentos de la realidad, siempre 'en comunicación', a través de la crítica intersubjetiva entre pares” (Bellón 2003: 11). Su trabajo de campo (cualitativo), basado en el "análisis de contenido e interpretación" de las obras publicadas por nueve investigadores mexicanos de la comunicación miembros del sNi sobre esta práctica, y entrevistas semiestructuradas con todos ellos, sistemáticamente analizadas, la lleva a plantear, entre otras varias agudas "interpretaciones", que:

Para la totalidad de los investigadores entrevistados el diálogo entre parescompetidores es valorado de manera positiva, pues favorece la construcción de conocimiento por el obvio enriquecimiento del objeto de estudio y el aumento del aporte empírico debido al trabajo conjunto. Sin embargo, se reconoce también la "escasez de lectura", la "falta de discusión permanente" y de "foros para el debate", la "desinformación", el "malinchismo", el "aislamiento", los temas u objetos como "propiedad de los investigadores", la "competencia en mala lid", la "personalización de las críticas", además de que el campo tiende al "envejecimiento" y es "centralista", entre otros. Al respecto, se rescata una aparente contradicción: el diálogo entre pares es valorado, pero no en la misma medida que la crítica y la evaluación de los productos de investigación (Bellón 2003: XIII). 
En otro estudio reciente del campo académico, también realizado como tesis de maestría pero con un enfoque totalmente diferente (bibliometría), el ya citado de Karla Ramírez (2003), se encuentra un punto de confluencia interesante, resultado de un análisis de citas aplicado a tres revistas mexicanas del campo, incluida Comunicación y Sociedad:

El análisis de citas permite registrar el uso del conocimiento científico implícito en los proyectos de investigación, trazar la influencia de una idea o captar la relevancia de un artículo u obra dentro de una comunidad científica. En el presente estudio, al cuantificar las citas emitidas, sólo aquellas que aparecían más de tres veces, dentro de los 184 productos seleccionados, se obtuvo un total de 125 referencias a autores nacionales e internacionales [de las cuales] el $76.8 \%$ son hechas a obras o artículos de autores extranjeros y sólo un 23.2\% a nacionales (Ramírez 2003).

Se trataba, con este recuento, precisamente de apreciar el grado en que se manifiestan en las publicaciones, por una parte, la dependencia intelectual o la "síntesis entre la metodología norteamericana y la reflexión europea" (Orozco 1997: 126-127) y por otra, los "referentes comunes" entre las revistas, que resultaron "casi nulos" (apenas La distinción de Pierre Bourdieu, La constitución de la sociedad de Anthony Giddens y Teoría de la acción comunicativa de Jürgen Habermas aparecieron con alguna frecuencia en las tres revistas). En medio de un gran desbalance (tres a uno) entre los autores extranjeros y los mexicanos citados en más de tres textos en las tres publicaciones, Ramírez encontró que "el equilibro se presenta en Comunicación y Sociedad, donde $51.06 \%$ son referencias a extranjeros y el restante a nacionales, siendo el sociólogo inglés Anthony Giddens y el filósofo español-latinoamericano Jesús Martín Barbero los más citados" (Ramírez 2003). Pero el dato que más interesa destacar aquí es el referido a las citas a autores nacionales:

[...] en relación con las referencias a obras o autores nacionales encontradas (un total de 28 citas), no se distinguen coincidencias entre las publicaciones, aun cuando sí es clara la forma en que se citan. En este sentido, entre las citas obtenidas de Comunicación y Sociedad (23 citas) se distinguen artículos y obras de miembros del Programa Cultura y de la UAMXochimilco [...] De igual forma, entre las citas localizadas en Comunicación y Sociedad se aprecia cómo los productores adscritos al DECS de la 
Universidad de Guadalajara se citan entre ellos constantemente, mas no en forma recíproca (Ramírez 2003).

Si bien la contribución explícita -objetivada mediante el análisis de citas de Ramírez-, al "diálogo entre pares-competidores" o bien a "la crítica y la evaluación de los productos de investigación" -en términos de la "vigilancia colectiva" de Bellón-, es mayor en Comunicación y Sociedad que la encontrada en otras revistas mexicanas, puede muy bien cerrarse este trabajo con una cita más de la primera de estas "nuevas" investigadoras de nuestro campo académico:

Finalmente, la escasa comunicación académica observada entre los productores nacionales -las citas entre ellos son realmente mínimas- obliga a pensar en un distanciamiento a nivel cognoscitivo. Es decir, la multiplicación de intereses reflejada en tópicos y enfoques teórico-metodológicos delinea un campo académico de la investigación de la comunicación en México fragmentado, sin encuentros, confrontaciones, ni cooperación, sin condiciones, como señalara Rosengren (1993), para "la acumulación de conocimientos necesaria para fortalecer al campo" o, si se quiere, como comentaba en su momento Gerbner (1983) "ejemplo de vitalidad" por la efervescencia galopante en que se encuentra (Ramírez 2003).

\section{REFERENCIAS BIBLIOGRÁFICAS}

Aceves González, Francisco de Jesús (1993) "Publicaciones sobre comunicación en México: las palabras errantes", Revista Mexicana de Comunicación, núm. 31, México, pp. 7-15.

Arredondo Ramírez, Pablo (1997) "La génesis de un espacio académico. Notas sobre la construcción del Centro de Estudios de la Información y la Comunicación de la Universidad de Guadalajara", Comunicación y Sociedad, núm. 30, Guadalajara, pp. 13-26.

Bellón CÁrdenAs, Raquel Elizabeth (2003) Construcción de conocimiento científico en comunicación: de la objetividad a la interpretación, tesis de maestría en Comunicación. México: Universidad Iberoamericana.

Clark Burton, R. (1992) El sistema de educación superior. Una visión comparativa de la organización académica. México: Nueva Imagen/Universidad Futura/Uam Azcapotzalco. 
Comunicación y Sociedad, núm. 30 (1997) “Presentación”, Guadalajara, pp. 7-12.

Crane, Diana (1972) Invisible Colleges. Diffusion of knowledge in scientific communities. Chicago y Londres: The University of Chicago Press.

Fuentes Navarro, Raúl (1990) "Cuadernos de Comunicación y la constitución de un campo académico y profesional en México (1975-1990)", Cuadernos de Comunicación, núm. 100, México, pp. 9-16.

(1994) "Las publicaciones académicas y la institucionalización del estudio de la comunicación en México", Comunicación y Sociedad, núms. 22/23, Guadalajara, pp. 71-102.

(1996a) "Un acercamiento bibliométrico a la configuración cognoscitiva del campo académico de la comunicación en México", Comunicación y Sociedad, núm. 27, Guadalajara, pp. 11-42.

(1996b) La investigación de la comunicación en México. Sistematización documental 1986-1994. Guadalajara: ITESo/Universidad de Guadalajara.

(1998a) "Décimo cumpleaños de la Revista Mexicana de Comunicación”, Revista Mexicana de Comunicación, núm. 56, México, pp. 7-10.

(1998b) La emergencia de un campo académico: continuidad utópica y estructuración científica de la investigación de la comunicación en México. Guadalajara: ITESo/Universidad de Guadalajara.

(2000) "La investigación latinoamericana sobre medios masivos e industrias culturales y la comunicación”, en Franz Portugal B. (comp.), La investigación en comunicación en América Latina 1970-2000. Lima: Asociación Peruana de Facultades de Comunicación Social, pp. 288-296.

(2003) La investigación académica sobre comunicación en México. Sistematización documental 1995-2001. Guadalajara: ITESO.

GERBNER, George (1983) "The importance of being critical in our own's fashion. An epilogue", Ferment in the field, Journal of Communication, vol. 33, núm. 3, pp. 355-362.

Gómez Vargas, Héctor (2003) "Comentario a diez números del Anuario de Investigación de la Comunicación del CONEICC. Producción de conocimiento en un mundo desbordante", Anuario CONEICC de Investigación de la Comunicación $X$, México, pp. 9-16. 
Orozco Gómez, Guillermo (1997) La investigación de la comunicación dentro y fuera de América Latina, tendencias, perspectivas y desafíos del estudio de los medios. Argentina: Facultad de Periodismo y Comunicación Social, Universidad Nacional de la Plata.

(2000) "Desafíos de la investigación en comunicación para el nuevo milenio: un análisis a partir de diez revistas latinoamericanas”, Comunicação e Sociedade, núm. 33, São Paulo, pp. 53-66.

RamíReZ y Ramírez, Karla Margarita (2003) Destellos de la comunicación. La diseminación de conocimiento a través de las publicaciones académicas, tesis de maestría en Comunicación con especialidad en Difusión de la ciencia y la cultura. Guadalajara: ITESO.

Rosengren, Karl Erik (1993) "From field to frog ponds", The Future of the Field I, Journal of Communication, vol. 43, núm. 3.

SAINTOUT, Florencia (1998) "La perspectiva de la recepción de los medios masivos en la revista Comunicación y Sociedad. Una primera exploración”, Comunicación y Sociedad, núm. 32, Guadalajara, pp. 201-226.

SÁnchez RuIz, Enrique E. (1992) Medios de difusión y sociedad. Notas críticas y metodológicas. Guadalajara: CEIC Universidad de Guadalajara.

(1997) "Algunos retos para la investigación mexicana de comunicación. Una reflexión personal (en diálogo con Raúl Fuentes)”, Comunicación y Sociedad, núm. 30, Guadalajara, pp. 51-77.

SCHEFFLER Z., Eduardo (1998) "Razón y palabra: una publicación electrónica”, en Gómez Vargas (comp.), Realidad virtual. Redes de interacción y comunidades de investigación, col. Cuadernos de Mass Culturas, núm. 8. León: Universidad Iberoamericana-León, pp. 47-52.

Tovar Peña, Teresa (1997) "Una década de Comunicación y Sociedad”, Comunicación y Sociedad, Índice Acumulativo 1987-1997. Guadalajara: DECs Universidad de Guadalajara, pp. 7-18. 